\title{
Heat transfer enhancement from an inclined plate through a heat generating and variable porosity porous medium using nanofluids due to solar radiation
}

\author{
Sameh E. Ahmed ${ }^{1,2} \cdot$ Abdelraheem M. Aly $^{1,2} \cdot$ Z. A. S. Raizah ${ }^{1}$
}

(c) Springer Nature Switzerland AG 2019

\begin{abstract}
This work involves the numerical simulation of heat transfer enhancements by magnetohydrodynamic natural convection over an inclined, semi-infinite, non-reflecting, ideally transparent plate embedded in a variable-porosity, heat generating porous medium using nanofluid in the presence of solar radiation effect. The Brinkman extended Darcy model with the Forchheimer inertia term is assumed for the porous medium, while a single-phase model is used for the nanofluid model. Non-similar transformations are used to convert the governing equations to non-similar forms, and then a local non-similar technique is applied to solve the resulting system. Wide ranges of the governing parameter, namely, the heat loss coefficient, the Darcy number, the heat generation/absorption parameter, the magnetic field parameter, the nanoparticle volume fraction and the inclination angle are considered and a validation test is performed. The results show that among all the variable porosity models, M4 is found to be having a largest rate of fluid flow and temperature distributions, while M3 has the lowest. Also, the local Nusselt number is an increasing function of the heat loss coefficient and nanoparticle volume fraction.
\end{abstract}

Keywords Variable porosity $\cdot$ Nanofluids $\cdot$ Solar radiation $\cdot$ MHD $\cdot$ Heat generation/absorption

\section{List of symbols}

$a_{e} \quad$ Absorption coefficient

$a, b \quad$ Empirical constants

$c_{p} \quad$ Specific heat

$\mathrm{Da} \quad$ Darcy number

$F \quad$ Dimensionless stream function

g Gravitational acceleration

$G_{x} \quad$ Local Grashof number

$h \quad$ Heat transfer coefficient

$K \quad$ Permeability

M Magnetic field parameter

$\mathrm{Nu} \quad$ Nusselt number

Pr Prandtl number

$q^{\prime \prime} \quad$ Solar radiation flux

$Q \quad$ Heat generation/absorption parameter

Rc Heat loss coefficient

\section{$T \quad$ Temperature \\ $u, v \quad$ Velocity components \\ $x, y \quad$ Cartesian coordinates \\ $X, Y$ Dimensionless Cartesian coordinates}

\section{Greek symbols}

$\beta \quad$ Coefficient of thermal expansion

$\varepsilon \quad$ Porosity of the porous medium

$\varepsilon_{\infty} \quad$ Ambient porosity

$\beta_{0} \quad$ Strength of magnetic field

$\gamma \quad$ Ratio of nanolayer thickness to nanoparticle radius

$\sigma \quad$ Electrical conductivity

$\mu \quad$ Viscosity

$v \quad$ Kinematic viscosity

$\Phi \quad$ Inclination angle

$\phi \quad$ Solid volume fraction

$\Psi \quad$ Stream function

\footnotetext{
$\triangle$ Sameh E. Ahmed, sehassan@kku.edu.sa; Abdelraheem M. Aly, ababdallah@kku.edu.sa; Z. A. S. Raizah, zaalrazh@kku.edu.sa| ${ }^{1}$ Department of Mathematics, Faculty of Science, King Khalid University, Abha, Saudi Arabia. ${ }^{2}$ Department of Mathematics, Faculty of Science, South Valley University, Qena, Egypt.
} 
$\rho \quad$ Density

$\theta \quad$ Dimensionless temperature

$\zeta, \eta \quad$ Computational space coordinates

\section{Subscripts \\ nf Nanofluid \\ eff Effective \\ $f \quad$ Base fluid \\ p Nanoparticles}

\section{Introduction}

In the recent years, there are great aspects for modeling the non-Darcian transport through the porous media. Transport through the porous media has numerous practical applications including electronic cooling, thermal insulation, petroleum industries and geothermal systems. Vafai and his co-authors have presented early works on the porous media for the convective heat transfer. Vafai and Tien [1] presented the boundary and inertial effects in the fluid flow and convective heat transfer through the porous media. In addition, Vafai and Tien [2] presented the convective mass transfer through the porous media under the effects of the inertial effects. Vafai and Kim [3] obtained a solution for the flow in a porous channel using Brinkman-Forcheimer-extended Darcy model.

In the literature, there are several studies concern the enhanced models of the porous media. The enhanced models were used for simulating flow through packed and fluidized beds. Vafai [4] employed the model of the variable porosity media for the convective flow and heat transfer. Vafai et al. [5] investigated experimentally the heat transfer in the variable porosity media. Poulikakos and Renken [6] studied the effects of the variable porosity, flow inertia and Brinkman friction on the forced convection in a channel filled with porous media. Renken and Poulikakos [7] investigated the effects of the variable porosity on the forced convective heat transfer in the porous media. Hunt and Tien [8] studied the forced convection in fibrous media under the effects of thermal dispersion. Also, Hunt and Tien [9] investigated the non-Darcian convection in the cylindrical packed beds. The non-uniform porosity and thermal dispersion effects on the natural convection has been studied by Hsiao et al. [10]. Hong et al. [11] investigated the effect of the non-Darcian non-uniform porosity on the natural convection in porous media over the vertical-plate. They showed that the heat transfer is enhanced by the increase in dispersion parameter.

In the recent years, there are extensive studies on the hydromagnetic flows and heat transfer in porous media. The reason returns to their several engineering processes including casting, filtration of liquid metals, fusion control and cooling of nuclear reactors. Takhar and Bèg [12] summarized the effects of the magnetic field on the mixed convection flow over a vertical plate saturated with porous medium. Their results indicated that for the positive values of Eckert number, the magnetic field enhances the heat transfer. Chamkha [13] introduced the effects of Hall current on the hydromagnetic free convection on a vertical plate saturated with a porous medium. In addition, there are several studies focus on the natural convection due to the solar radiation. Fathalah and Elsayed [14] considered the natural convection flow due to the solar radiation in view of its possible application in the solar collectors. Chamkha [15] introduced a porous medium for the natural convection flow due to the solar radiation. In addition, Chamkha et al. [16] introduced a variable porosity for the natural convection due to the solar radiation.

Due to the low thermal conductivity of the traditional fluids compared to that of crystalline solids [17]. Hence, there are ideas to enhance thermal conductivity of the base fluid by adding small solid particles in the base fluid. This idea backs Maxwell's study [18]. Choi and Eastman [19] were firstly introduced the term of the nanofluid. Eastman et al. [20] used nanofluids to enhance heat transfer coefficients of 10-40\%. Xuan et al. [21] investigated the thermal conductivity of the nanofluids using the thermal Lattice Boltzmann model. Nield and Kuznetsov [22] studied the problem of Cheng and Minkowycz [23] for the natural convective flows over a vertical flat plate filled with nanofluid and saturated with a porous medium. Chamkha and Aly [24] studied the boundary layer flow of a nanofluid near a porous vertical plate under the effects of magnetic field and heat generation/absorption.

Bhatti et al. [25-30] introduced many analytical and numerical studies related to the enhancement heat transfer using nanofluid. Bhatti and Lu [25] introduced analytical study for heat on collision between hydro-elastic solitary waves. Sheikholeslami and Bhatti $[29,30]$ studied the nanofluid forced convection under the effects of Coulomb force in a porous semi-annulus in the presence of uniform magnetic field. In addition, using the nanofluids in investigation the heat transfer in cases of external and internal flows can be found in [31-35]. Here it should be mentioned that there are semi-analytical methods that can solve this problem [36].

On the other hand, by examinations the study that reported by Fathalah and Elsayed [14], it is found that they neglected the cases of variable porosity, inclined plate, heat generation effects, nanofluids and presence of magnetic field. Also, Chamkha [15] generalized the study of Fathalah and Elsayed [14] by considering the case of the porous media but he neglected the cases of the variable porosity, inclined plate, nanofluids, presence of the magnetic force and heat generation effects. 
Moreover, according to our knowledge, there are no published studies related to the effect of the solar radiation on the magnetohydrodynamic natural convection over an inclined plate embedded in a variable-porosity with nanofluid. Hence, the purpose of this study is to involve the numerical simulation of the heat transfer enhancements by the magnetohydrodynamic natural convection over an inclined, semi-infinite, ideally transparent plate embedded in a variable-porosity in the presence of the solar radiation effect. The Brinkman extended Darcy model with the Forchheimer inertia term is assumed for the porous medium, while a single-phase model is used for the nanofluid. The governing equations have been transformed to the non-similar forms using Non-similar transformations. Wide ranges of the governing parameter, namely, the heat loss coefficient, Darcy number, heat generation/absorption parameter, nanoparticle volume fraction, magnetic field parameter and an inclination angle are considered. The results show that among all of the variable porosity models, M4 is found to be having the largest rate of the fluid flow and temperature distributions, while M3 has the lowest. In addition, the local Nusselt number is an increasing function of the heat loss coefficient and nanoparticle volume fraction. A validation test is performed compare to the previous published result with well agreement.

\section{Problem assumptions and mathematical model}

Consider the boundary layer of the flow of magnetohydrodynamic nanofluid over a plate inclined with the angle $\Phi$ embedded in a porous medium. Figure 1 shows the physical model and Cartesian coordinates of the problem. It shows that the $x$-axis is along the plate and $y$-axis is normal to it. In the current simulation, the following assumptions are considered:

- The flow is laminar, two dimensional and steady.

- The nanofluid is viscous, incompressible and electrically conducting.

- The plate is inclined with angle $\Phi$, semi-infinite, nonreflecting, ideally transparent and non-absorbing.

- The intensity of the solar radiation flux is $q^{\prime \prime}$ and it penetrates the wall to be absorbed in a homogeneous nanofluid of the absorption coefficient $a_{e}$.

- The heat of the plate is lost to the surroundings by the heat transfer coefficient $R$.

- There is a heat source inside the flow domain with dimensional heat generation/absorption parameter $Q_{0}$.

- All times, there is a magnetic field of strength $\beta_{0}$ affects in the negative $y$ direction.

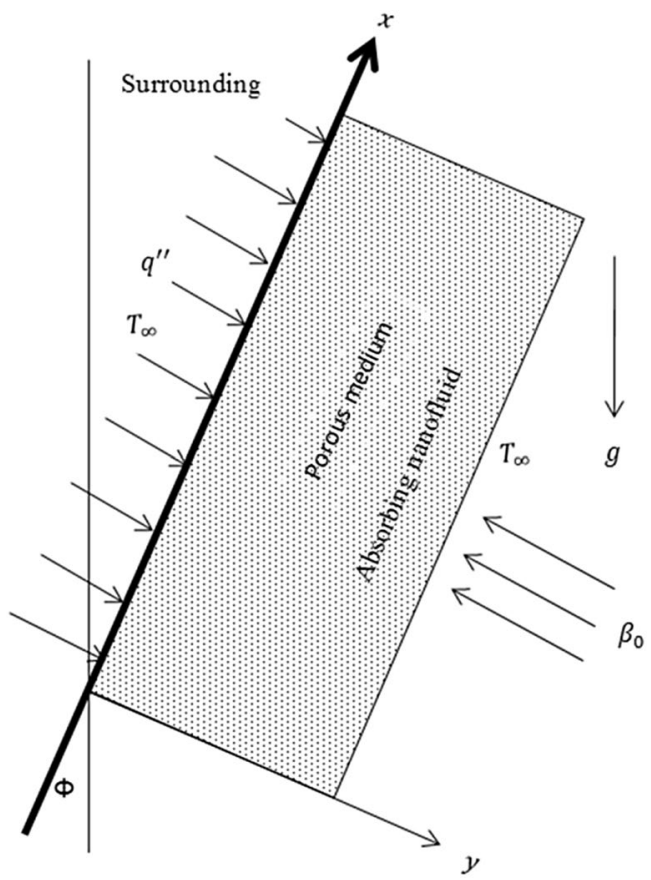

Fig. 1 Physical model of the problem and coordinates system

Table 1 Thermo-physical properties for the base fluid and the nanoparticles

\begin{tabular}{lll}
\hline Property & Water & Copper(Cu) \\
\hline$\rho$ & 997.1 & 8933 \\
$C_{p}$ & 4179 & 385 \\
$k$ & 0.613 & 401 \\
$\beta$ & $21 \times 10^{-5}$ & $1.67 \times 10^{-5}$ \\
$\sigma$ & 0.05 & $5.96 \times 10^{7}$ \\
\hline
\end{tabular}

- For the porous medium, we considered the Darcy model of Brinkman extended with the Forchheimer inertia term.

- The porous medium is isotropic and homogenous and the local thermal equilibrium model between the porous medium and the fluid is applied.

- The non-uniform porosity of the porous medium is considered, while the Joule heating and viscous dissipation effects are ignored.

- The single phase nanofluid model is applied and the LTEM (local thermal equilibrium model) between the fluid and suspended nanoparticle is applied.

- The base fluid is water with $\operatorname{Pr}=6.2$ at $20^{\circ} \mathrm{C}$ and copper is the nanoparticles.

- The nanofluid thermophysical properties are presented in Table 1. 
- The density of the fluid is approximated via Boussinesq approximation, and all other properties of the fluid are constants.

Taking into account all these assumptions, the continuity, momentum and energy equations can be written as (see Vafai and Tien [1] and Gebhart et al. [37])

$\frac{\partial u}{\partial x}+\frac{\partial v}{\partial y}=0$ where $\varepsilon$ is the porosity of the porous medium and in this study it is assumed to be a function of the Cartesian coordinates y as follows:

$\varepsilon(y)=\varepsilon_{\infty}\left(1+a \exp \left[\frac{-b y}{d_{p}}\right]\right)$

In Eq. (5) $\varepsilon_{\infty}$ is ambient porosity and $a$ and $b$ are empirical constants that depend on the ratio of the porous bed to

$u \frac{\partial u}{\partial x}+v \frac{\partial u}{\partial y}=v_{n f} C_{1} \frac{\partial^{2} u}{\partial y^{2}}-\frac{v_{n f}}{K(y)} u-C(y) u^{2}-\frac{\sigma_{n f} \beta_{0}^{2}}{\rho_{n f}} u+g \frac{(\rho \beta)_{n f}}{\rho_{n f}}\left(T-T_{\infty}\right) \cos \Phi$

$u \frac{\partial T}{\partial x}+v \frac{\partial T}{\partial y}=\frac{k_{\text {eff }}}{\left(\rho C_{p}\right)_{n f}} \frac{\partial^{2} T}{\partial y^{2}}+\frac{Q_{0}}{\left(\rho C_{p}\right)_{n f}}\left(T-T_{\infty}\right)+\frac{a_{e} q^{\prime \prime}}{\left(\rho C_{p}\right)_{n f}} \exp \left(-a_{e} y\right)$

Subjected to the following boundary conditions:

$$
\begin{aligned}
u(x, 0) & =v(x, 0)=0, k_{\text {eff }} \frac{\partial T(x, 0)}{\partial y}=R\left(T(x, y)-T_{\infty}\right), \\
u(x, \infty) & =0, T(x, \infty)=T_{\infty}
\end{aligned}
$$

In Eqs. (1)-(4), $u$ and $v$ are the velocity components in the $x$ - and $y$-directions, $g$ is the gravity acceleration, $T$ is the temperature, $K$ is the permeability, $v$ is the kinematic viscosity, $\sigma$ is the electrical conductivity, $\rho$ is the density, $\beta$ is the thermal expansion, $C_{p}$ is the specific heat, and the subscripts $n f$, eff refer to the nanofluid and effective, respectively.

\subsection{Properties of the porous medium}

Different models of variable porosity are summarized by Alazmi and Vafai [38] and in this study, it is needed to compare among these models to present a comprehensive study. The following table displays the forms of these models in which the porosity is variable:

\begin{tabular}{llcc}
\hline Model & $C_{1}$ & $\frac{1}{K(y)}$ & $C(y)$ \\
\hline$M_{1}$ & 1 & $\frac{150(1-\varepsilon)^{2}}{\varepsilon^{3} d_{p}^{2}}$ & $\frac{1.75(1-\varepsilon)}{\varepsilon^{3} d_{p}}$ \\
$M_{2}$ & $\frac{1}{\varepsilon}$ & $\frac{150(1-\varepsilon)^{2}}{\varepsilon^{3} d_{p}^{2}}$ & $\frac{1.75(1-\varepsilon)}{\varepsilon^{3} d_{p}}$ \\
$M_{3}$ & $\frac{1}{\varepsilon}$ & $\frac{150(1-\varepsilon)^{2}}{\varepsilon^{3} d_{p}^{2}}$ & $\frac{1.75(1-\varepsilon)}{\varepsilon^{2} d_{p}}$ \\
$M_{4}$ & 1 & $\frac{150(1-\varepsilon)^{2}}{\varepsilon^{2} d_{p}^{2}}$ & $\frac{1.75(1-\varepsilon)}{\varepsilon^{2} d_{p}}$ \\
\hline
\end{tabular}

particle. In addition, Eq. (5) is an approximate representation of the experimental data reported by Benenati and Brosilow [39] for their study on void fraction distribution in packed beds.

To give a good approximation to the data reported by Benenati and Brosilow [39] for a particle diameter of $4 \mathrm{~mm}$, we chose the values of $\varepsilon_{\infty}, a$, and $c$ employed in the present work as $0.5,0.98$, and 1 , respectively.

\subsection{Nanofluid properties}

Here, a single-phase nanofluid model is employed to the present simulation. In this model the nanoparticle volume fractions is taken as independent variable and the thermophysical properties are taken as function of it. Following Daniel Makinde [40], the nanofluid density, the thermal diffusivity, the dynamic viscosity, the thermal expansion, the heat capacitance, the electrical conductivity, and the thermal conductivity of nanofluid are given as follows:

$\rho_{n f}=\phi \rho_{p}+(1-\phi) \rho_{f}$

$\alpha_{n f}=\frac{k_{e f f}}{\left(\rho C_{p}\right)_{n f}}$

$\mu_{n f}=\frac{\mu_{f}}{(1-\phi)^{2.5}}$

$(\rho \beta)_{n f}=\phi(\rho \beta)_{p}+(1-\phi)(\rho \beta)_{f}$

$(\rho C)_{n f}=\phi(\rho C)_{p}+(1-\phi)(\rho C)_{f}$ 
$\sigma_{n f}=\sigma_{f}\left[1+\frac{3\left[\frac{\sigma_{s}}{\sigma_{f}}-1\right] \phi}{\left[\frac{\sigma_{s}}{\sigma_{f}}+2\right]-\left[\frac{\sigma_{s}}{\sigma_{f}}-1\right] \phi}\right]$

$k_{n f}=k_{f} \frac{\left(k_{p}+2 k_{f}\right)-2 \phi(1+\gamma)^{3}\left(k_{f}-k_{p}\right)}{\left(k_{p}+2 k_{f}\right)+\phi(1+\gamma)^{3}\left(k_{f}-k_{p}\right)}$

In the previous equations, $\phi$ is the solid volume fraction and $\gamma$ is the ratio of nanolayer thickness to nanoparticle radius and thereby accounts for the size dependent nature of thermal conductivity of nanofluids. Here, a nanolayer thickness of $1 \mathrm{~nm}$ is considered which corresponds to $\gamma=0.1$. Also, the subscripts $f, n f$, and $p$ refer to the base fluid, nanofluid and nanoparticles, respectively.

\section{Solution of the problem}

In this section, non-similar solutions are used to the governing equations. These solutions required to introduce the following transformations as well as the stream functions as:

$$
\begin{aligned}
& \frac{k_{e f f}}{k_{f}} \frac{\left(\rho C_{p}\right)_{f}}{\left(\rho C_{p}\right)_{n f}} \theta^{\prime \prime}+\operatorname{Pr}\left[4 F \theta^{\prime}-F^{\prime} \theta-4 \xi\left[F^{\prime} \frac{\partial \theta}{\partial \xi}-\frac{\partial F}{\partial \xi} \theta^{\prime}\right]\right] \\
& \quad+25 \xi^{\frac{1}{2}} \frac{\left(\rho C_{p}\right)_{f}}{\left(\rho C_{p}\right)_{n f}} Q \theta-5 \xi^{\frac{1}{4}} \frac{\left(\rho C_{p}\right)_{f}}{\left(\rho C_{p}\right)_{n f}} \exp \left(-5 \xi^{\frac{1}{4}} \eta\right)=0
\end{aligned}
$$

where $D a=a_{e} d_{p}$ is the Darcy number, $M^{2}=\sigma_{f} \beta_{0}^{2} / a_{e} \mu_{f}$ is the magnetic field parameter and $Q=\frac{Q_{0}}{\alpha_{f} a_{e}^{2}\left(\rho C_{p}\right)_{f}}$ is the heat generation absorption parameter, $\operatorname{Pr}=\frac{v_{f}}{\alpha_{f}}$ is the Prandtl parameter. Moreover, the non-similar form of the boundary conditions (Eq. 4) is:

$F(\xi, 0)=F^{\prime}(\xi, 0)=0, \frac{k_{e f f}}{k_{f}} \theta^{\prime}(\xi, 0)=5 R c \xi^{\frac{1}{4}} \theta(\xi, 0)$

$F^{\prime}(\xi, \infty)=0, \theta(\xi, \infty)=0$

In Eq. (18), $R c=\frac{R}{k_{f} a_{e}}$ is the heat loss coefficient. It should be mentioned that wide ranges for $\xi$ and $R c$ are considered in this study, where, $\xi$ ranging from 0.0001 to 10 and $R c$ is ranging from 0 to 10 . These ranges are due to cover the wide range of the absorption coefficient $a_{e}$ as well as the

$$
\begin{aligned}
& \Psi=v_{f} G_{x} F(\xi, \eta), \quad T=T_{\infty}-\frac{5 x q^{\prime \prime}}{k_{f} G_{x}} \theta(\xi, \eta), \quad \xi=\frac{G_{x}}{G_{a}^{5}}, \quad \eta=\frac{y G_{x}}{5 x}, \quad G_{x}=5\left[\frac{g \beta_{f} q^{\prime \prime} x^{4}}{5 k_{f} v_{f}^{2}}\right]^{\frac{1}{5}} \\
& G_{a}=5\left[\frac{g \beta_{f} q^{\prime \prime}}{5 k_{f} v_{f}^{2} a_{e}^{4}}\right]^{\frac{1}{5}}, \quad u=\frac{\partial \Psi}{\partial y}, \quad v=-\frac{\partial \Psi}{\partial x} .
\end{aligned}
$$

where $G_{x}$ is the local Grashof number based on the distance along the plate $x$ and $G_{a}$ is the $G_{a}$ is the Grashof number based on the effective absorption coefficient $a_{e}$. Here, the velocity components take the forms:

$u=\frac{v_{f} G_{x}^{2}}{5 x} F^{\prime}$

$v=-v_{f} \frac{G_{x}}{5 x}\left[4 F+4 \xi \frac{\partial F}{\partial \xi}-\eta \frac{\partial F}{\partial \eta}\right]$

Substituting Eqs. (13)-(15) into Eqs. (1)-(3), the continuity equation is satisfied identically, while the momentum and energy equations take the following forms: measurements of Dorsey for the absorption of radiation in water layers with different thickness as stated by Cooper [41]. Note, the absorption coefficient $a_{e}$ depends on the type of absorbing fluid and its degree of colouring or contamination. Also, as mentioned by Fathalah and Elsayed [14], the Dorsey's data was analyzed using the Beer's law which takes the form:

$q_{a b s}^{\prime \prime}=q^{\prime \prime}\left(1-\exp \left(-a_{e} y\right)\right)$

Justification of the ranges of $a_{e}$ and the radiation flux intensity $q^{\prime \prime}$ are introduced in the studies presented by Cooper [29] and Fathalah and Elsayed [14].

$$
\begin{aligned}
& \frac{v_{n f}}{v_{f}} F^{\prime \prime \prime}-3 F^{\prime 2}+4 F F^{\prime \prime}+4 \xi\left[F^{\prime \prime} \frac{\partial F}{\partial \xi}-F^{\prime} \frac{\partial F^{\prime}}{\partial \xi}\right]-25 \xi^{\frac{1}{2}} \frac{v_{n f}}{v_{f}}\left[\frac{150(1-\varepsilon)^{2}}{D a^{2} \varepsilon^{3}}+M^{2} \frac{\sigma_{n f}}{\sigma_{f}} \frac{\mu_{f}}{\mu_{n f}}\right] F^{\prime} \\
& -5 \xi^{\frac{5}{4}}\left[\frac{1.75(1-\varepsilon) G_{a}^{5}}{\varepsilon^{3} D a}\right] F^{\prime 2}-\frac{(\rho \beta)_{n f}}{\rho_{n f} \beta_{f}} \theta \cos \Phi=0
\end{aligned}
$$




\section{Local Nusselt number}

The local Nusselt number based on the absorption parameter is defined by:

$N u=\frac{h}{k_{f} a_{e}}$

where $h$ is the heat transfer coefficient which based on the maximum temperature [14] and it is given by:

$h=\frac{q_{u}^{\prime \prime}}{T_{\max }-T_{\infty}}$

Here $q_{u}^{\prime \prime}$ is the useful heat gain collected by the absorbing fluid and it can be expressed as:

$q_{u}^{\prime \prime}=q^{\prime \prime}-R\left[T_{p}-T_{\infty}\right]$

where $T_{p}$ is the plate temperature. Substituting Eq. (22) into Eq. (21), the following form for $h$ is obtained:

$h=\frac{q^{\prime \prime}}{T_{\max }-T_{\infty}}-R \frac{T_{p}-T_{\infty}}{T_{\max }-T_{\infty}}$

Inserting Eq. (23) in Eq. (20) and using the non-similar transformations (Eq. 13), the local Nusselt number takes the form:

$N u=-\frac{1}{\theta_{\max }}\left[\frac{1}{5 \xi^{1 / 4}}+R_{c} \theta_{p}\right]$

\section{Numerical solutions and validation}

The non-similar Eqs. (16) and (17) with the boundary conditions (18) are solved numerically using the MATLAB function bvp4c. All the details of this method are found in Ahmed and Mahdy [42] and Mahdy and Ahmed [43]. The step size is set as $\Delta \eta=0.05$ and the convergence criterion of the solution required that the relative difference between two successive iterations be $10^{-5}$. The accuracy of this method is checked by valuable comparisons with previously published results in special cases of the current study. Figure 2 shows a comparison of the velocity profiles and temperature distributions for different values of the loss coefficient $R c$ with those obtained by obtained by Fathalah and Elsayed [14] at $\operatorname{Pr}=6.5, D a=\infty, Q=0, M=0, \phi=0 \%, \Phi=0, \xi=0.1$. It is observed that the present results are much closed to those of Fathalah and Elsayed [14].

\section{Results and discussion}

In order to get a clear physical insight, a set of graphical figures is presented and discussed here. The obtained results are plotted in terms of velocity profiles, temperature distributions and local Nusselt number; those are presented for wide ranges of the governing parameters. As stated later, the range of the loss coefficient $R c$ is chosen between 0 and 10 and this selection to cover the high velocities of wind. Also, the range of heat generation/absorption parameter $Q$ is chosen between -2 and 3 to represent both the heat generation and the heat absorption cases inside the porous medium. Regarding the ranges of the inclination angle $\Phi(0 \leq \Phi \leq \pi / 6)$, magnetic field parameter $\mathrm{M}(0 \leq \mathrm{M} \leq 5)$ and Darcy number $\mathrm{Da}(0.5 \leq \mathrm{Da} \leq 5)$, those are chosen according to the studies reported by Chamkha et al. [16] and Chamkha [15]. The value of $\operatorname{Pr}=6.7$ is selected to represent water at $20{ }^{\circ} \mathrm{C}$ and the range of the nanoparticle volume fraction $\phi(0 \% \leq \phi \leq 6 \%)$ to be suitable for the nanofluid thermal conductivity correlation. Additionally, the value of $G a=1.25$ is chosen to correspond the radiation flux of $900 \mathrm{~W} / \mathrm{m}^{2}$.

Figure 2 shows the distributions of the porosity of the porous medium along the boundary layer. It is found that the range of $\varepsilon$ considered in this study is between 0.98 at the wall and 0.5 far away from the wall. This figure helps the reader in understanding the behavior of velocity and temperature. In addition $\mathrm{Yu}$ and Choi [44] presented a modification for the Maxwell model [45] by including the ratio of nanolayer thickness to nanoparticle radius. Figure 2 displays a comparison between these two models. It is observed that the thermal conductivity obtained by model [44] is greater than that of [45] (Figs. 3, 4).

Figures 5 and 6 show the velocity profiles and temperature distributions for the different models used for the variable-porosity porous medium. The results indicated that among all these models, M4 gives the high behavior of the velocity and nanofluid temperature and $M 3$ gives the low one. Also, the profiles of M2 and M1 are closed to each other but $\mathrm{M} 2$ gives the high rate comparing with M1. These behaviors are attributed to the resistance force resulting from the elements of the porous medium that damps the convective flow and this force in M4 is the smallest one compared with the other model, see Table 1.

The influence of the heat loss coefficient $R c$ on the velocity profiles, temperature distributions and local Nusselt number are depicted in Figs. 7, 8 and 9, respectively. It is clear that in the absence of solar radiation, the maximum value of the temperature occurs at the wall but when the radiation effect is considered, the maximum temperature 
Fig. 2 comparison of the present results (left) and those obtained by Fathalah and Elsayed [14] (right) at

$\operatorname{Pr}=6.5, D a=\infty, Q=0, M=0$ $\phi=0 \%, \Phi=0, \xi=0.1$.
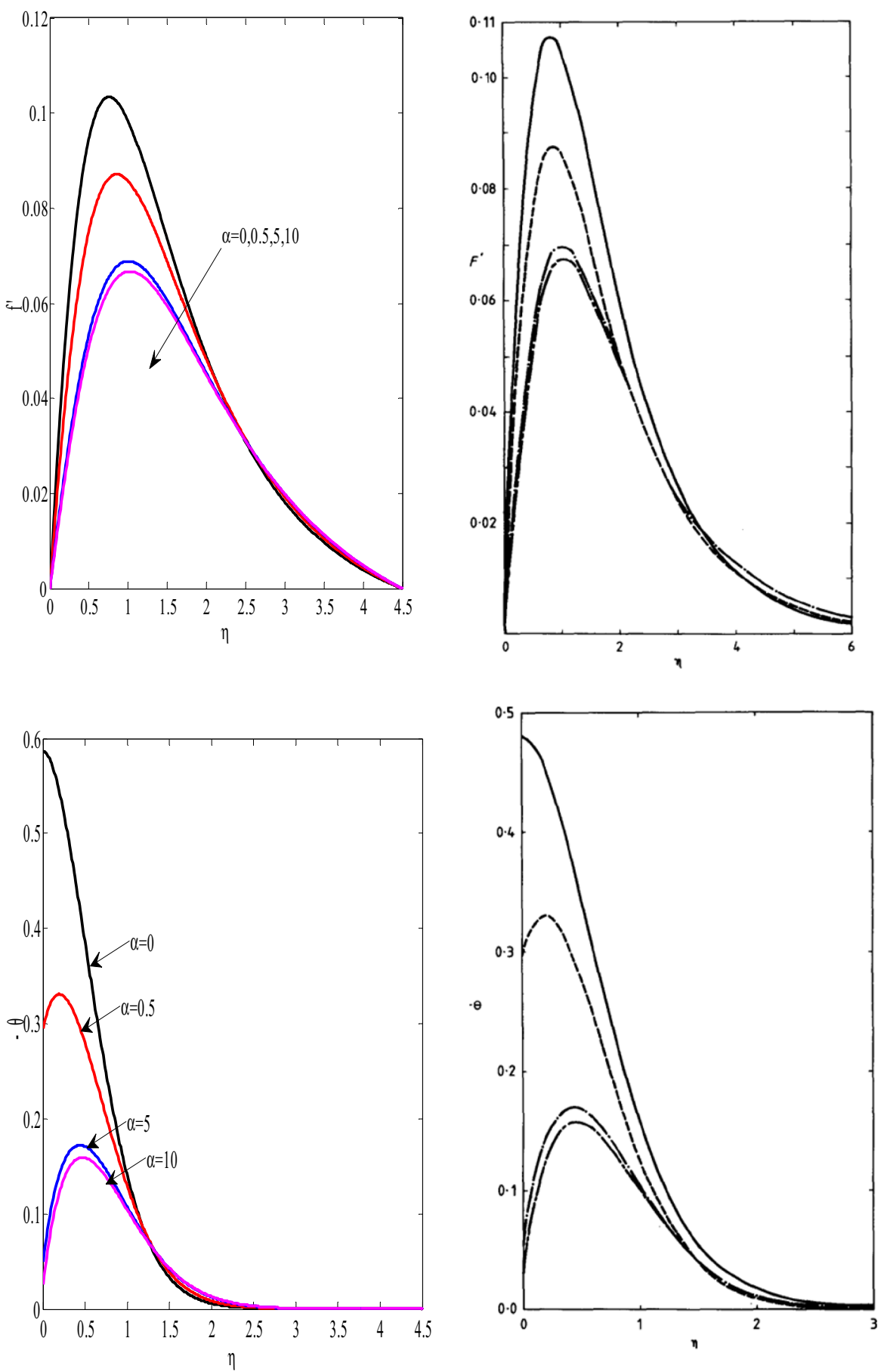

occurs inside the nanofluid not on the wall. The physical explanations of this effect are due to absorption of the radiation by the nanofluid and the ideally transparent wall can only heated via the absorbing nanofluid. Also, this description interprets the overshot in the velocity behavior near the wall in which occurs the maximum temperature.
In addition, it is observed that the increase in Rc causes a reduction in the velocity profiles and the maximum temperature. Consequently the local Nusselt number is enhanced for all models as Rc increases.

Figures 10 and 11 determine the velocity profiles and temperature distributions for the variations of the 


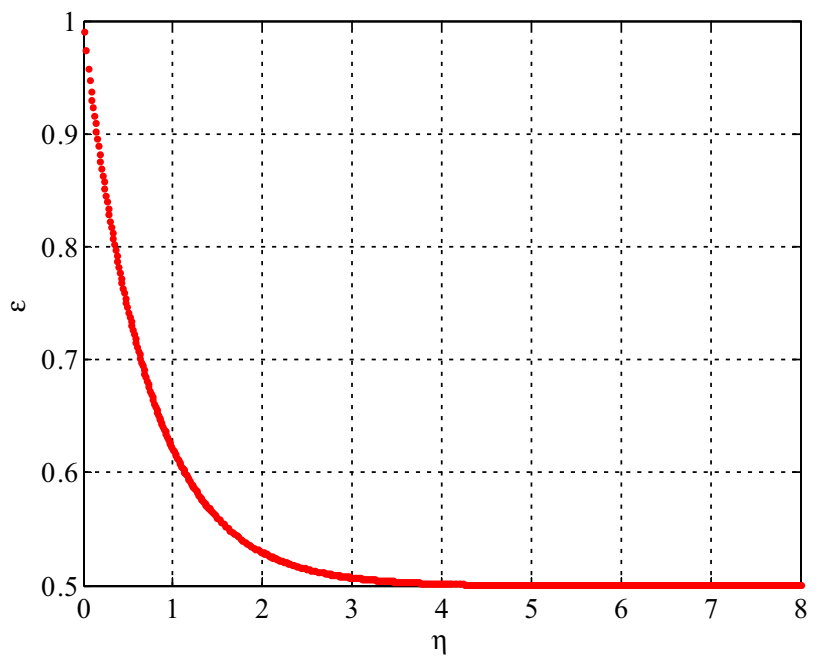

Fig. 3 Distributions of the porosity through the boundary layer at $\xi=0.1, D a=2$

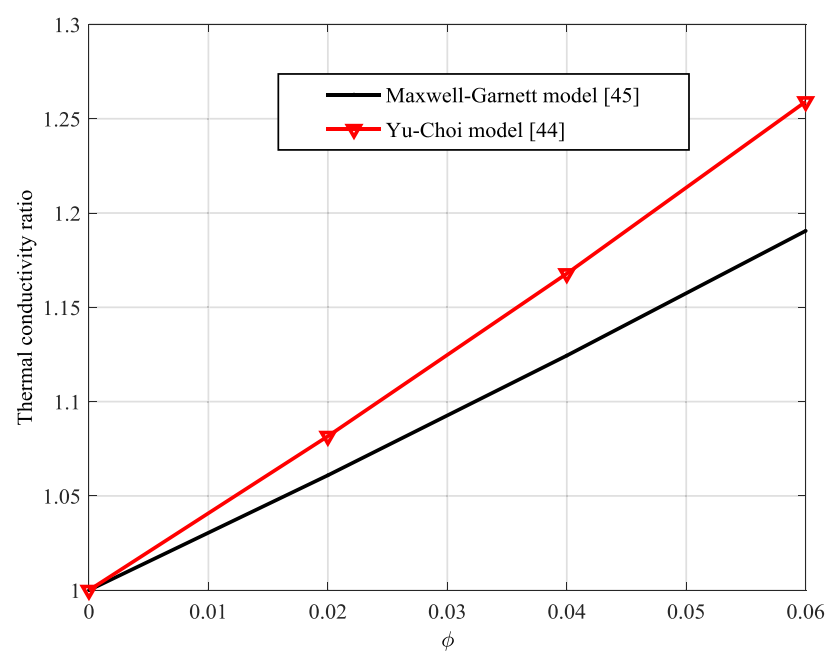

Fig. 4 Comparison of the present model (Yu and Choi model [44]) and Maxwell-Garnett model [45] for the considered range of $\phi$.

nanoparticle volume fraction $\phi$. It of noted that the increase in $\phi$ reduces both the velocity and temperature. This is because the viscosity of the nanofluid increases by addition the nanoparticles (i.e. increase of $\phi$ ) which slowdown the velocity. Like the effect of $\phi$ on the velocity profiles, the presence of magnetic field acts as a retarding force to the fluid flow. As it can be seen in Fig. 12, the increase in $\mathrm{M}$ reduces the profiles of $\mathrm{F}^{\prime}$. However, the maximum temperature increases, slightly, as $M$ increases, as it can be observed in Fig. 13. This is because of the Lorentz

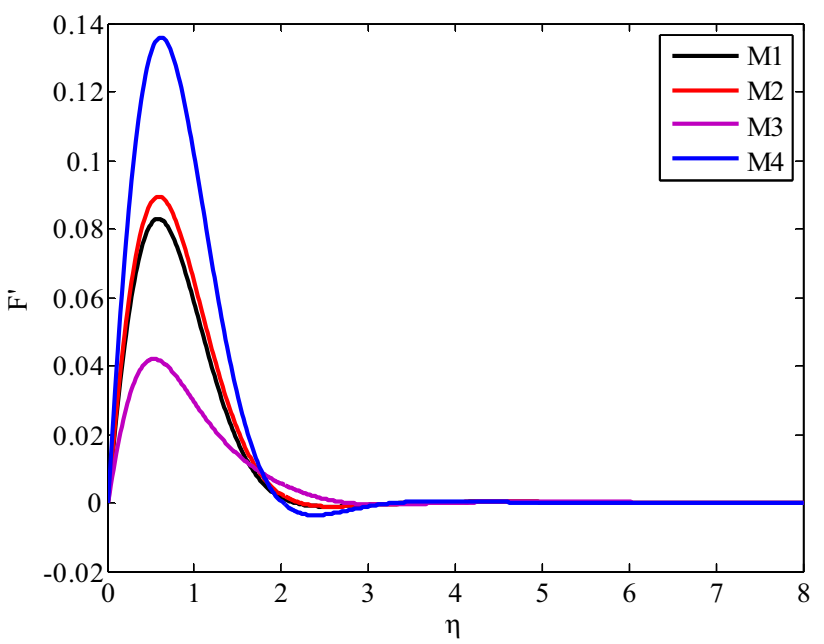

Fig. 5 Velocity profiles for different models at $R c=5, G a=1.25, D a=5, Q=1, M=1, \phi=2 \%, \Phi=\frac{\pi}{6}, \xi=0.1$

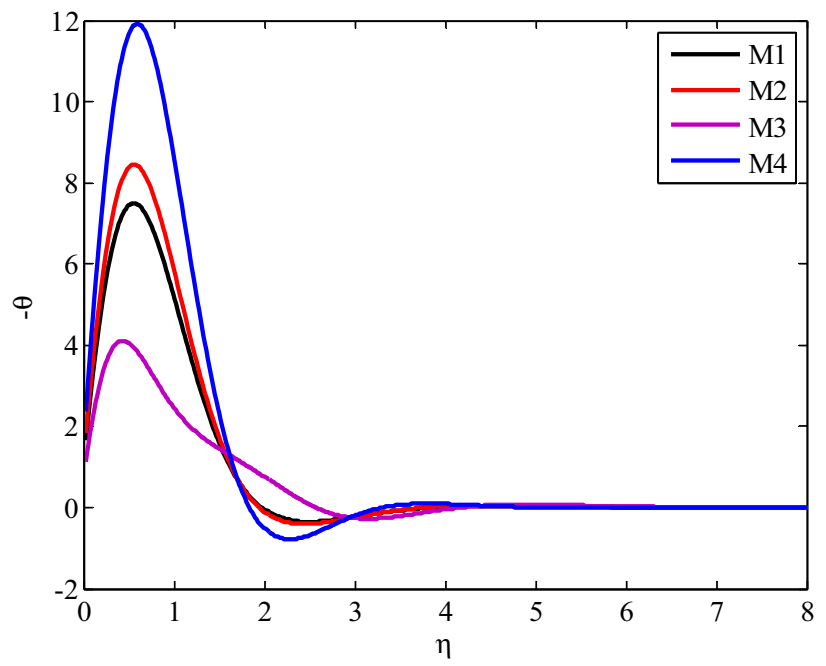

Fig. 6 Temperature distributions for different models at $R c=5, G a=1.25, D a=5, Q=1, M=1, \phi=2 \%, \Phi=\frac{\pi}{6}, \xi=0.1$

force resulting from the presence of magnetic field which heated up the nanofluid.

Figures 14 and 15 illustrate the profiles of velocity and temperature for different values of the heat generation/ absorption parameter $\mathrm{Q}$. The results revealed that both the velocity and temperature increase gradually as $Q$ increases indicating to a good natural convection by increasing $\mathrm{Q}$. This behavior is anticipated because the increase in $Q$ means an additional heat in the nanofluid which makes the fluid flow rapidly. 


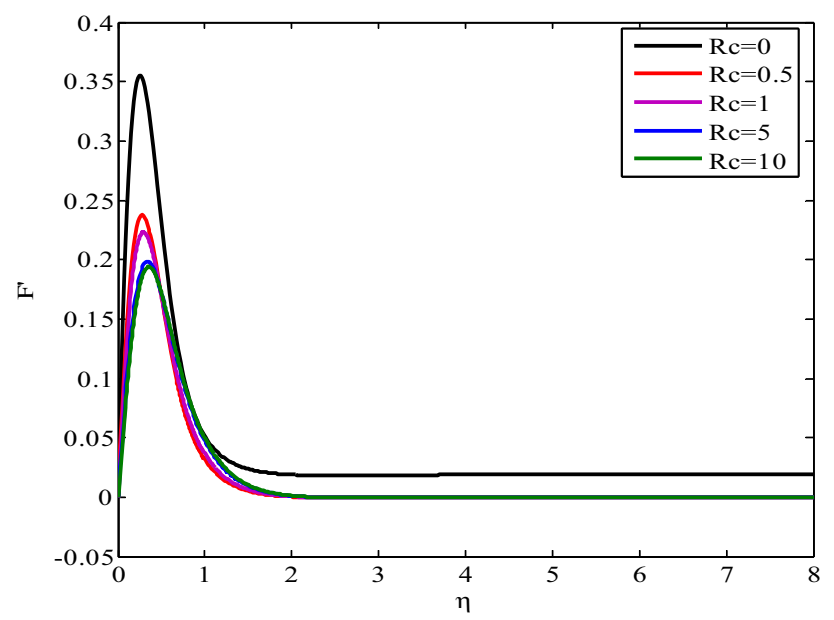

Fig. 7 Velocity profiles for different values of $R c$ at $M 1, G a=1.25, D a=2, Q=1, M=1, \phi=2 \%, \Phi=\frac{\pi}{6}, \xi=0.1$

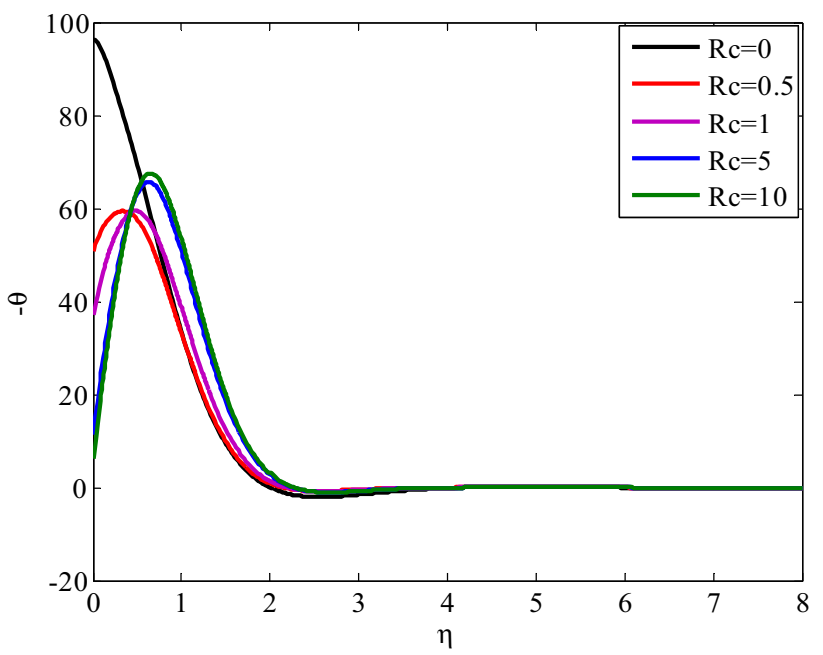

Fig. 8 Temperature distributions for different values of $R c$ at $M 1, G a=1.25, D a=2, Q=1, M=1, \phi=2 \%, \Phi=\frac{\pi}{6}, \xi=0.1$

The effects of the inclination angle of the plate on the velocity and temperature are examined with the help of Figs. 16 and 17. It is found that the increase in the inclination angle causes an increase in the thermal buoyancy effect and hence the velocity profiles and maximum temperature are enhanced. This behavior is observed for $\Phi \leq \pi / 4$, however more increase in $\Phi$ leads to reduce the velocity and temperature profiles.

Figure 18 focuses on the effect of variation of Darcy number $\mathrm{Da}$ on the velocity profiles. It observed that the

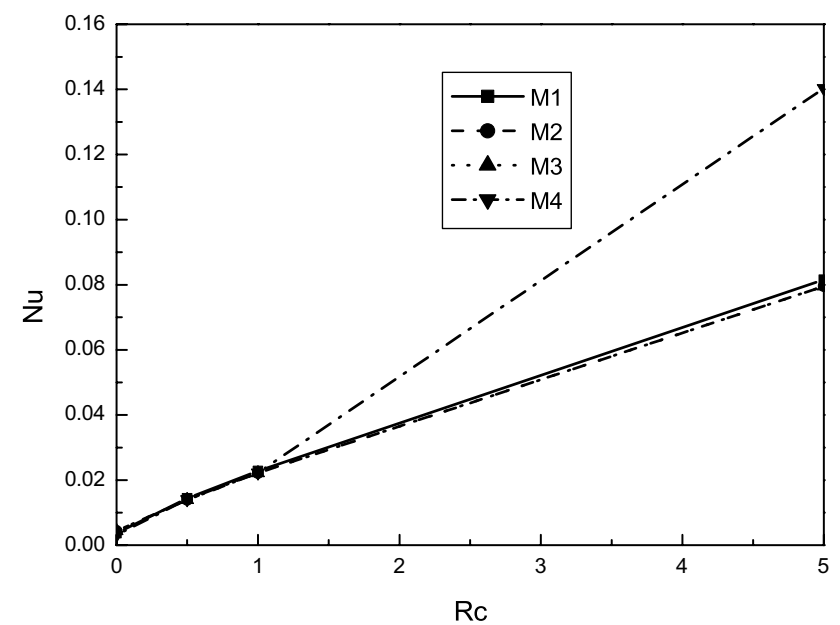

Fig. 9 Effects of the heat loss coefficient on the local Nusselt number for different models at $D a=2, G a=1.25, Q=1, M=1, \phi=2 \%, \Phi=\frac{\pi}{6}, \xi=0.1$

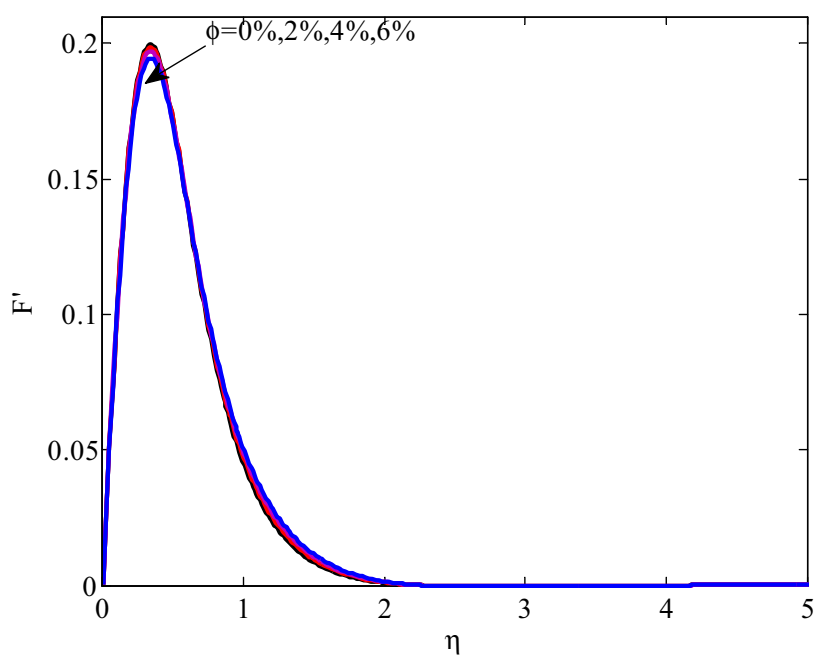

Fig. 10 Velocity profiles for different values of $\phi$ at $M 1, R c=5, G a=1.25, D a=2, Q=1, M=1, \Phi=\frac{\pi}{6}, \xi=0.1$

behavior of $F^{\prime}$ at the values of $D a<1$ larger than those of $D a \geq 1$. Also, the behaviors correspond the values of $D a \geq 1$ are closed to each other. On the other hand the increase in Da causes a decrease in the behaviors of velocity pointing an enhancement in rate of heat transfer. To explain these behaviors, it should be mentioned to the definition of $\mathrm{Da}$ $\left(D a=a_{e} d_{p}\right)$. It depends on the diameter of the porous medium's element. Therefore the increase in $D a$ means the increase in $d_{p}$ which brings much resistance to the flow and hence $F^{\prime}$ decreases. 


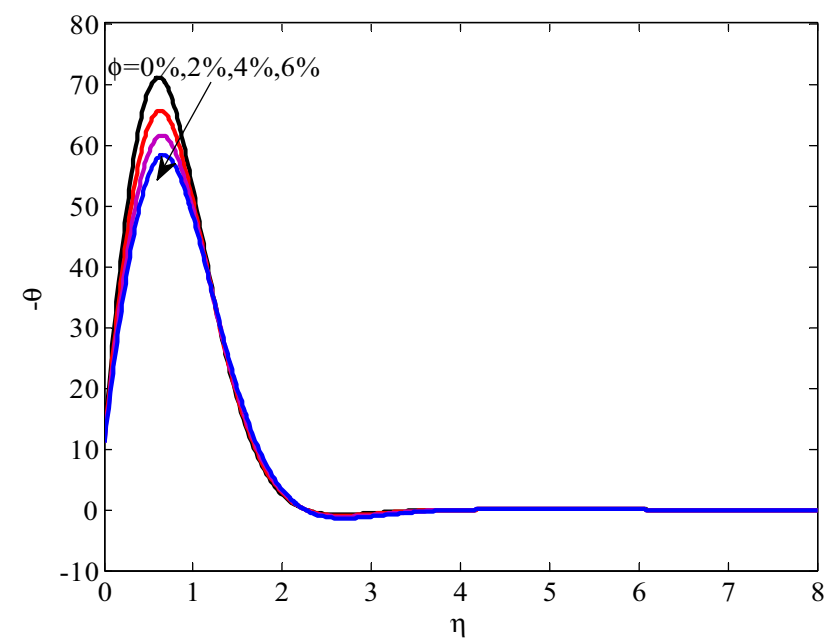

Fig. 11 Temperature distributions for different values of $\phi$ at $M 1, R c=5, G a=1.25, D a=2, Q=1, M=1, \Phi=\frac{\pi}{6}, \xi=0.1$

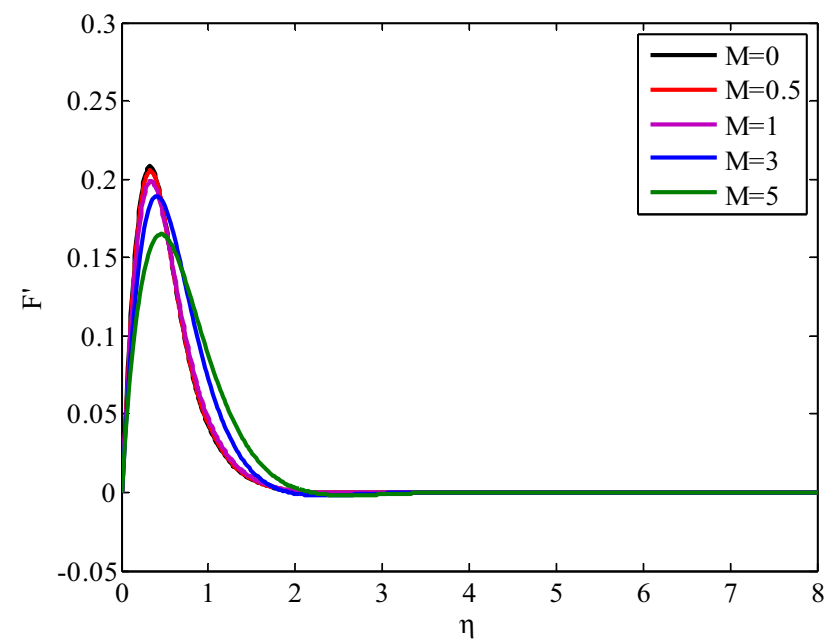

Fig. 12 Velocity profiles for different values of $M$ at $M 1, R c=5, G a=1.25, D a=2, Q=1, M=1, \phi=2 \%, \Phi=\frac{\pi}{6}, \xi=0.1$

\section{Conclusions}

Steady-state natural convection and heat transfer over an inclined plate through a heat generating and variable porosity porous medium in the presence of solar radiation using nanofluids have been, numerically, investigated in this study. The used nanofluid consists of Cu-nanoparticles suspended in water at $20^{\circ} \mathrm{C}$. The Brinkman extended Darcy model was considered to simulate the porous

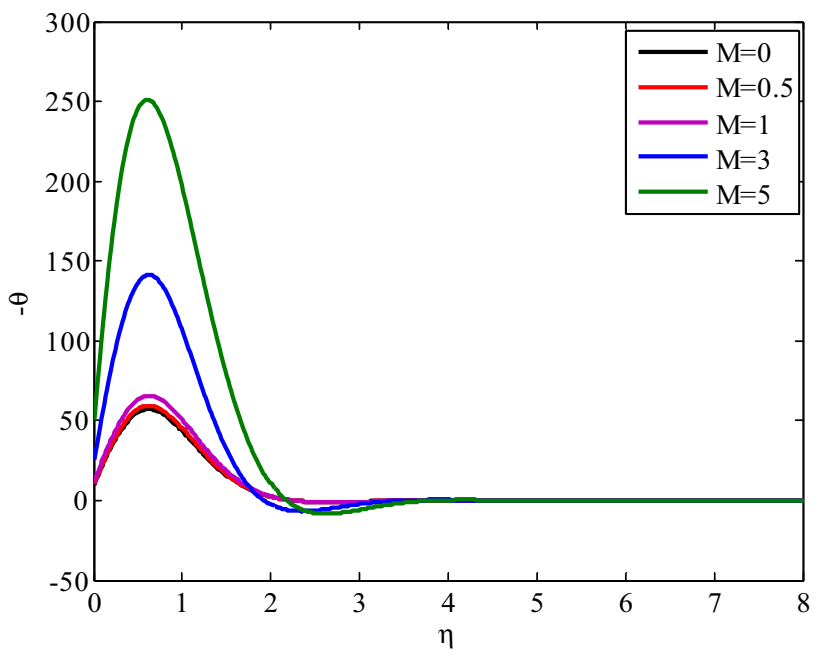

Fig. 13 Temperature distributions for different values of $M$ at $M 1, R c=5, G a=1.25, D a=2, Q=1, \phi=2 \%, \Phi=\frac{\pi}{6}, \xi=0.1$

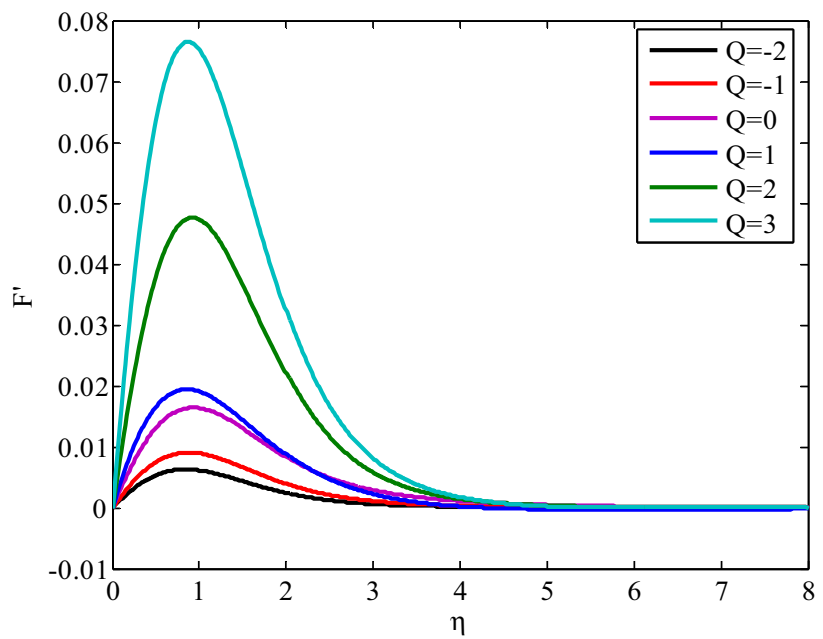

Fig. 14 Velocity profiles for different values of $Q$ at $M 1, R c=5, G a=1.25, D a=2, Q=1, M=1, \phi=2 \%, \Phi=\frac{\pi}{6}, \xi=0.001$

medium and four models for the variable porosity were taken into account. Based on the findings of this study, it can be concluded that:

- M4 gives the largest values of the velocity and temperature while M3 gives the lowest values.

- The increase in the loss coefficient decreases the velocity profiles while it increases the local Nusselt number. 


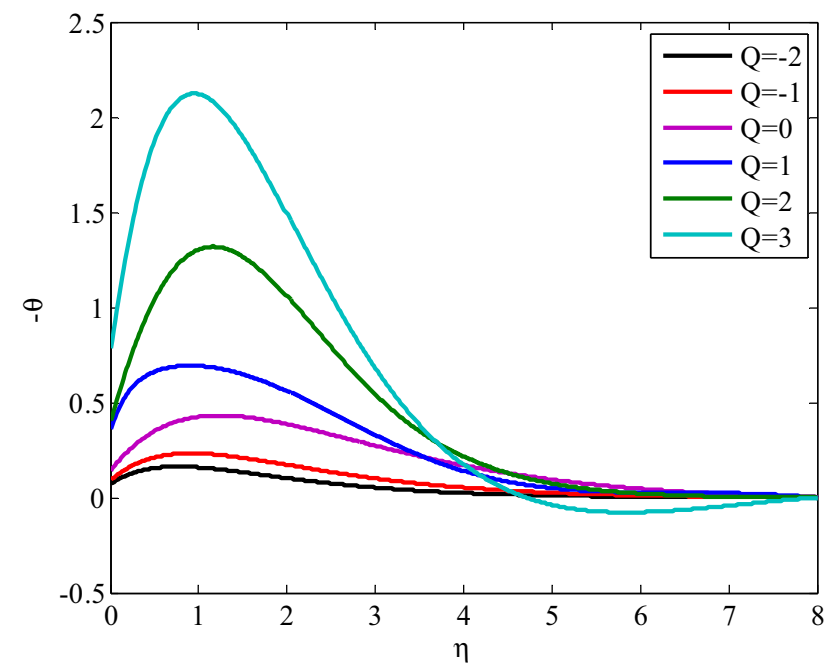

Fig. 15 Temperature distributions for different values of $Q$ at $M 1, R c=5, G a=1.25, D a=2, Q=1, M=1, \phi=2 \%, \Phi=\frac{\pi}{6}, \xi=0.001$

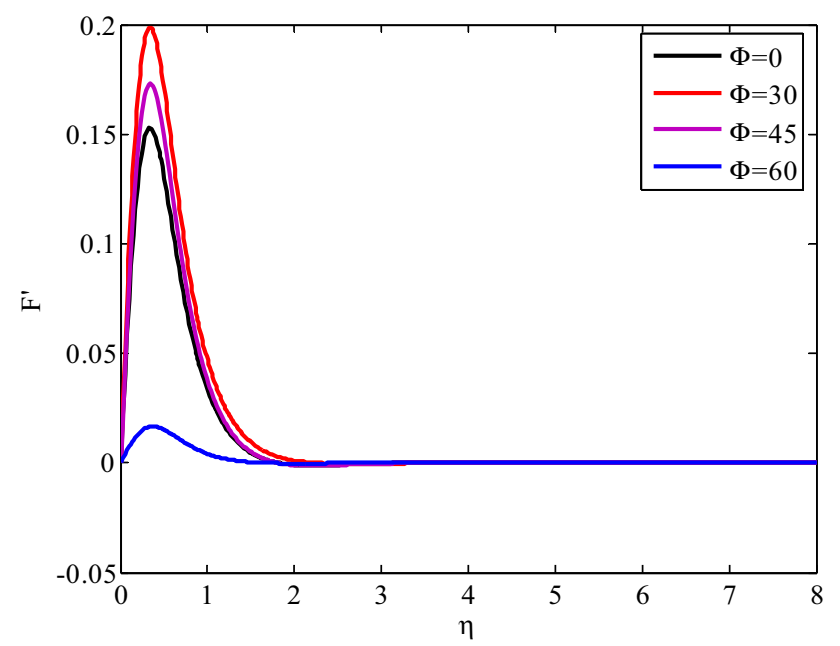

Fig. 16 Velocity profiles for different values of $\Phi$ at $M 1, G a=1.25, D a=2, Q=1, M=1, \phi=2 \%, \Phi=\frac{\pi}{6}, \xi=0.1$

- The increase in the nanoparticles volume fraction causes a reduction in the profiles of velocity and temperature while it enhances the local Nusselt number.

- As the magnetic parameter increases, the velocity profiles decreases but the local temperature is enhanced.

- The local Nusselt number is an increasing function of Darcy number, heat generation/absorption parameter, inclination angle and magnetic field parameter.

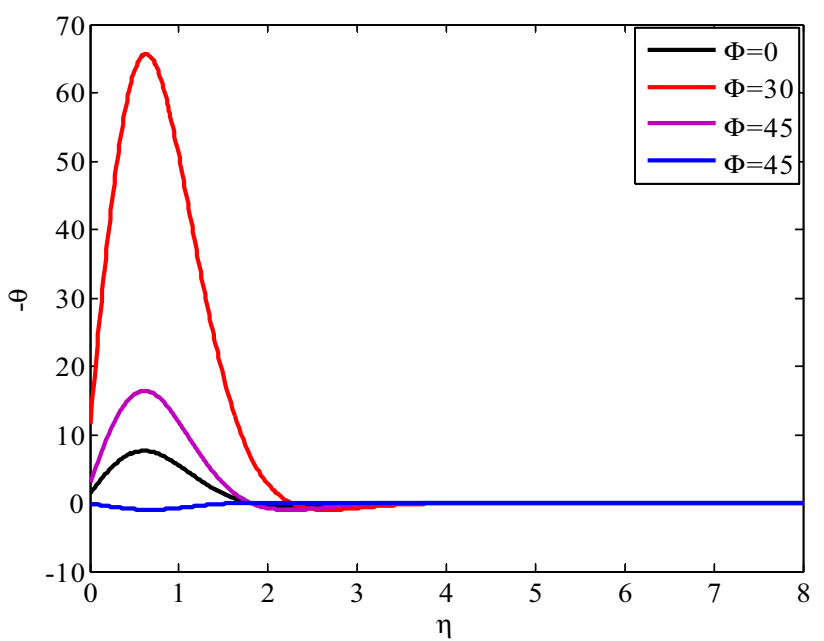

Fig. 17 Velocity profiles for different values of $\Phi$ at $M 1, G a=1.25, D a=2, Q=1, M=1, \phi=2 \%, \Phi=\frac{\pi}{6}, \xi=0.1$

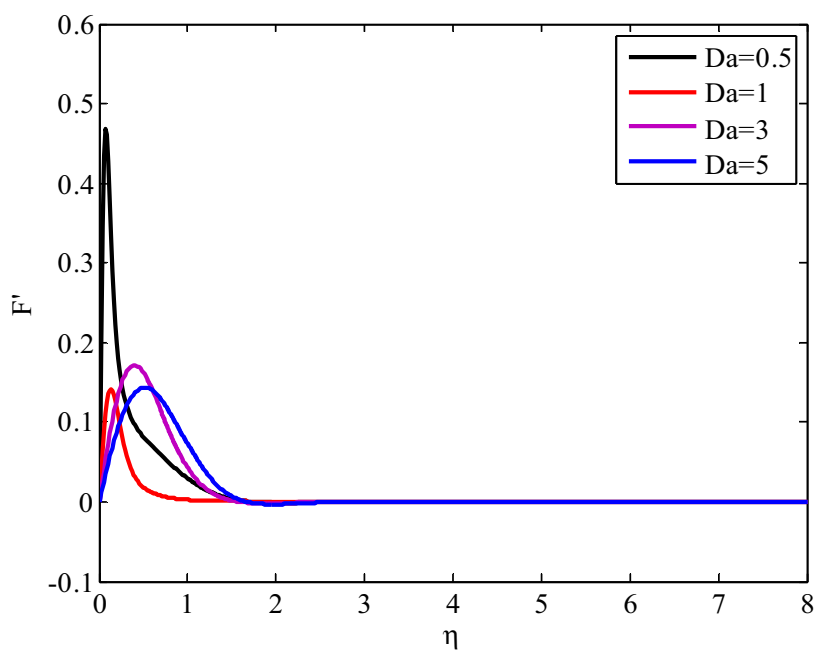

Fig. 18 Velocity profiles for different values of $D a$ at $M 1, G a=1.25, D a=2, Q=1, M=1, \phi=2 \%, \Phi=\frac{\pi}{6}, \xi=0.2$.

Acknowledgements The authors would like to express their gratitude to King Khalid University, Saudi Arabia, for providing administrative and technical support.

\section{Compliance with ethical standards}

Conflict of interest The authors declare that they have no competing interests. 


\section{References}

1. Vafai K, Tien CL (1981) Boundary and inertia effects on flow and heat transfer in porous media. Int J Heat Mass Transf 24:195-203

2. Vafai K, Tien CL (1982) Boundary and inertia effects on convective mass transfer in porous media. Int J Heat Mass Transf 25:1183-1190

3. Vafai K, Kim SJ (1989) Forced convection in a channel filled with a porous medium: an exact solution. ASME J Heat Transf 111:1103-1106

4. Vafai K (1984) Convective flow and heat transfer in variable porosity media. J Fluid Mech 147:233-259

5. Vafai K, Alkire RL, Tien CL (1985) An experimental investigation of heat transfer in variable porosity media. ASME J Heat Transf 107:642-647

6. Poulikakos D, Renken K (1987) Forced convection in a channel filled with porous medium, including the effects of flow inertia, variable porosity and Brinkman friction. ASME J Heat Transf 109:880-888

7. Renken K, Poulikakos D (1988) Experiment and analysis of forced convective heat transport in a packed bed of spheres. Int J Heat Mass Transf 25:1399-1408

8. Hunt ML, Tien CL (1988) Effects of thermal dispersion on forced convection in fibrous media. Int J Heat Mass Transf 31:301-309

9. Hunt ML, Tien CL (1988) Non-Darcian convection in cylindrical packed beds. ASME J Heat Transf 110:378-384

10. Hsiao SW, Cheng P, Chen CK (1992) Non-uniform porosity and thermal dispersion effects on natural convection about a heated horizontal cylinder in an enclosed porous medium. Int J Heat Mass Transf 35:3407-3418

11. Hong JT, Yamada Y, Tien CL (1987) Effect of non-Darcian nonuniform porosity on vertical-plate natural convection in porous media. ASME J Heat Transf 109:356-362

12. Takhar HS, Bèg OA (1997) Effects of transverse magnetic field, Prandtl number and Reynolds number on non-Darcy mixed convective flow of an incompressible viscous fluid past a porous vertical flat plate in a saturated porous medium. Int J Energy Res 21:87-100

13. Chamkha AJ (1997) MHD-free convection from a vertical plate embedded in a thermally stratified porous medium with hall effects. Appl Math Model 21:603-609

14. Fathalah KA, Elsayed MM (1980) Natural convection due to solar radiation over a non-absorbing plate with and without heat losses. Int J Heat Fluid Flow 2:41-45

15. Chamkha AJ (1997) Solar radiation assisted natural convection in uniform porous medium supported by a vertical flat plate. ASME J Heat Transf 119:35-43

16. Chamkha AJ, Issa C, Khanafer K (2002) Natural convection from an inclined plate embedded in a variable porosity porous medium due to solar radiation. Int J Therm Sci 41:73-81

17. Büyük Öğüt E (2009) Natural convection of water-based nanofluids in an inclined enclosure with a heat source. Int J Therm Sci 48:2063-2073

18. Maxwell JC (ed) (2010) A treatise on electricity and magnetism. Cambridge University Press, Cambridge, pp 21-30

19. Choi SUS (1995) Enhancing thermal conductivity of fluids with nanoparticles. In: Siginer DA, Wang HP (eds) Developments and applications of non-Newtonian flows, vol 66. ASME, New York, pp 99-105

20. Eastman JA, Choi SUS, Li S, Yu W, Thompson LJ (2001) Anomalously increased effective thermal conductivities of ethylene glycol-based nanofluids containing copper nanoparticles. Appl Phys Lett 78:718-720
21. Xuan Y, Yu K, Li Q (2004) Investigation on flow and heat transfer of nanofluids by the thermal Lattice Boltzmann model. Prog Comput Fluid Dyn Int J 5:13-19

22. Nield DA, Kuznetsov AV (2009) The Cheng-Minkowycz problem for natural convective boundary-layer flow in a porous medium saturated by a nanofluid. Int J Heat Mass Transf 52:5792-5795

23. Cheng P, Minkowycz WJ (1977) Free convection about a vertical flat plate embedded in a porous medium with application to heat transfer from a dike. J Geophys Res 82:2040-2044

24. Chamkha AJ, Aly AM (2010) MHD free convection flow of a nanofluid past a vertical plate in the presence of heat generation or absorption effects. Chem Eng Commun 198:425-441

25. Bhatti MM, Lu DQ (2019) Analytical study of the head-on collision process between hydroelastic solitary waves in the presence of a uniform current. Symmetry 11(3):333

26. Bhatti MM, Rashidi MM (2016) Effects of thermo-diffusion and thermal radiation on Williamson nanofluid over a porous shrinking/stretching sheet. J Mol Liq 221:567-573

27. Bhatti MM, Abbas T, Rashidi MM, Ali ME-S, Yang Z (2016) Entropy generation on MHD Eyring-Powell nanofluid through a permeable stretching surface. Entropy 18(6):224

28. Bhatti MM, Zeeshan A, Ellahi R, Shit GC (2018) Mathematical modeling of heat and mass transfer effects on MHD peristaltic propulsion of two-phase flow through a Darcy-Brinkman-Forchheimer porous medium. Adv Powder Technol 29(5):1189-1197

29. Sheikholeslami M, Bhatti MM (2017) Active method for nanofluid heat transfer enhancement by means of EHD. Int J Heat Mass Transf 109:115-122

30. Sheikholeslami M, Bhatti MM (2017) Forced convection of nanofluid in presence of constant magnetic field considering shape effects of nanoparticles. Int J Heat Mass Transf 111:1039-1049

31. Ahmed SE, Elshehabey HM (2018) Buoyancy-driven flow of nanofluids in an inclined enclosure containing an adiabatic obstacle with heat generation/absorption: effects of periodic thermal conditions. Int J Heat Mass Transf 124:58-73

32. Ahmed SE, Raizah ZAS (2018) Natural convection flow of nanofluids in a composite system with variable-porosity media. J Thermophys Heat Transf 32(2):495-502

33. Hussain S, Ahmed SE, Saleem F (2018) Impact of periodic magnetic field on entropy generation and mixed convection. J Thermophys Heat Transf 32(4):999-1012

34. Ahmed SE, Hussein AK, Mansour MA, Raizah ZA, Zhang X (2018) MHD mixed convection in trapezoidal enclosures filled with micropolar nanofluids. Nanosci Technol Int J 9(4):343-372

35. Mansour MA, Mohamed RA, Abd-Elaziz MM, Ahmed SE (2010) Numerical simulation of mixed convection flows in a square liddriven cavity partially heated from below using nanofluid. Int Commun Heat Mass Transf 37(10):1504-1512

36. Chamekh M, Elzaki TM (2018) Explicit solution for some generalized fluids in laminar flow with slip boundary conditions. J Math Comput Sci 18:272-281

37. Gebhart B, Jaluria Y, Mahajan RL, Sammakia B (1988) Buoyancy induced flows and transport, Hemisphere, New York, p 914

38. Alazmi B, Vafai K (1999) Analysis of variants within the porous media transport models. J Heat Transf 122(2):303-326

39. Benenati RF, Brosilow CB (1962) Void fraction distribution in beds of spheres. AIChE J 8(3):359-361

40. Daniel Makinde O (2013) Effects of viscous dissipation and Newtonian heating on boundary-layer flow of nanofluids over a flat plate. Int J Numer Methods Heat Fluid Flow 23:1291-1303

41. Cooper PI (1972) Some factors affecting the absorption of solar radiation in solar stills. Sol Energy 13:373-381

42. Ahmed SE, Mahdy A (2016) Unsteady MHD double diffusive convection in the stagnation region of an impulsively rotating 
sphere in the presence of thermal radiation effect. J Taiwan Inst Chem Eng 58:173-180

43. Mahdy A, Ahmed SE (2015) Thermosolutal Marangoni boundary layer magnetohydrodynamic flow with the Soret and Dufour effects past a vertical flat plate. Eng Sci Technol Int J 18(1):24-31

44. Yu W, Choi SUS (2003) The role of interfacial layers in the enhanced thermal conductivity of nanofluids: a renovated Maxwell model. J Nanopart Res 5(1):167-171
45. Garnett JCM, Larmor J (1904) XII. Colours in metal glasses and in metallic films. Philos Trans R Soc Lond Ser A Contain Pap Math Phys Character 203(359-371):385-420

Publisher's Note Springer Nature remains neutral with regard to jurisdictional claims in published maps and institutional affiliations. 